\title{
Sensitivity of ice flow to uncertainty in flow law parameters in an idealized one-dimensional geometry Supplementary material
}

Maria Zeitz ${ }^{1,2}$, Anders Levermann ${ }^{1,2,3}$, and Ricarda Winkelmann ${ }^{1,2}$

${ }^{1}$ Potsdam Institute for Climate Impact Research (PIK), Member of the Leibniz Association, P.O. Box 6012 03, 14412 Potsdam, Germany

${ }^{2}$ University of Potsdam, Institute of Physics and Astronomy, Karl-Liebknecht-Str. 24-25, 14476 Potsdam, Germany

${ }^{3}$ LDEO, Columbia University, New York, USA.

Correspondence: Maria Zeitz (maria.zeitz@ pik-potsdam.de), Ricarda Winkelmann (ricarda.winkelmann@ pik-potsdam.de) 


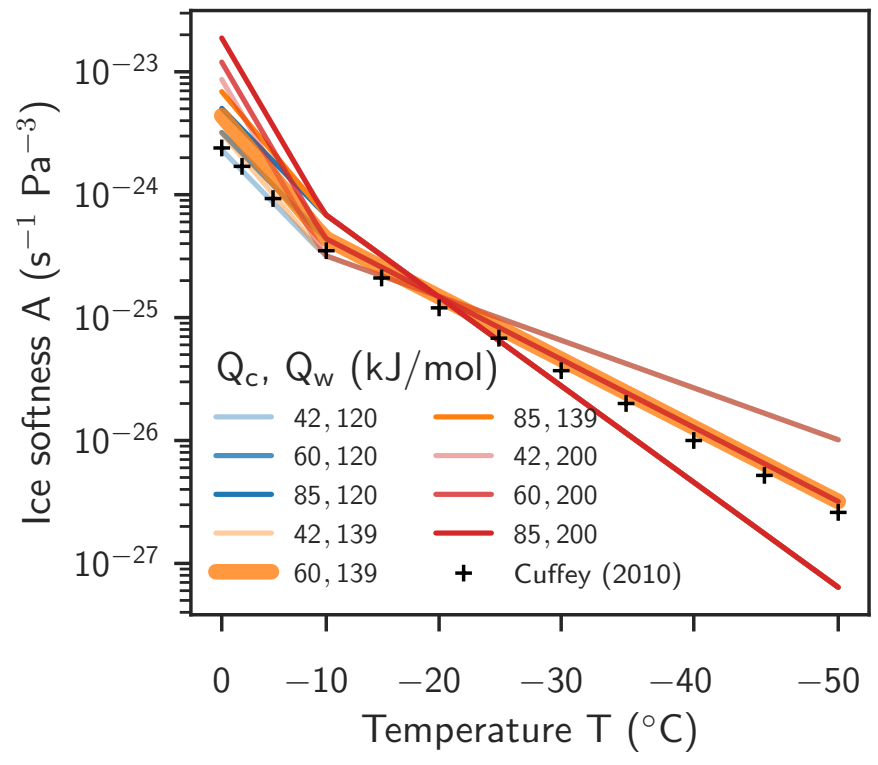

Figure S1. Effect of activation energy parameters on the temperature dependence of the softness $A$. The temperature dependence of the ice softness $A$ is usually shown in an Arrhenius plot, where the softness is shown on a semi-log scale over the inverse temperature. Two parameters for the activation energy $Q_{\mathrm{c}}$ and $Q_{\mathrm{w}}$ for $T \leq-10^{\circ} \mathrm{C}$ and $T>-10^{\circ} \mathrm{C}$ parametrize the relationship of ice softness to pressure adjusted temperature. Here the softness is fixed at a reference temperature of $T=-20^{\circ} \mathrm{C}$. The softness at cold temperatures depends only on the choice of $Q_{\mathrm{c}}$. Softness at pressure melting point is most sensitive for variations in the $Q_{\mathrm{w}}$, but varies slightly with $Q_{\mathrm{c}}$. At pressure melting point the softness increases 8 -fold between the limits of parameter combinations, from $-47 \%$ to $+335 \%$ compared to standard parameters. For comparison we show the textbook values of the softness for different temperatures (black crosses) (?). 

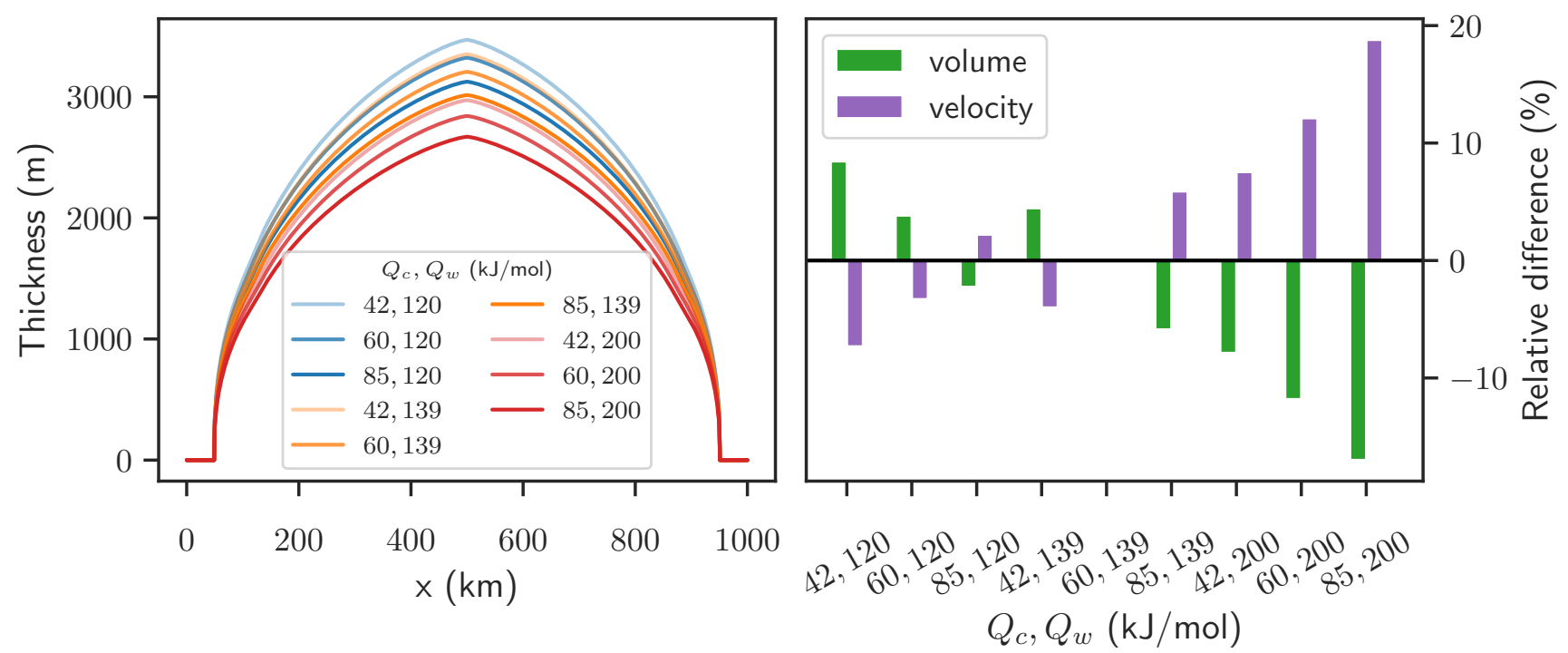

Figure S2. Effect of flow parameters on equilibrium state without warming with fixed accumulation rates Cross sections of equilibrium states (a) relative difference of average velocities and equilibrium volumes compared to the reference state with standard parameters (b) for parameter combinations of $Q_{\mathrm{w}}$ and $Q_{\mathrm{c}}$. The accumulation rate is fixed at $a=0.5 \mathrm{~m} / \mathrm{yr}$ for all parameter combinations. 


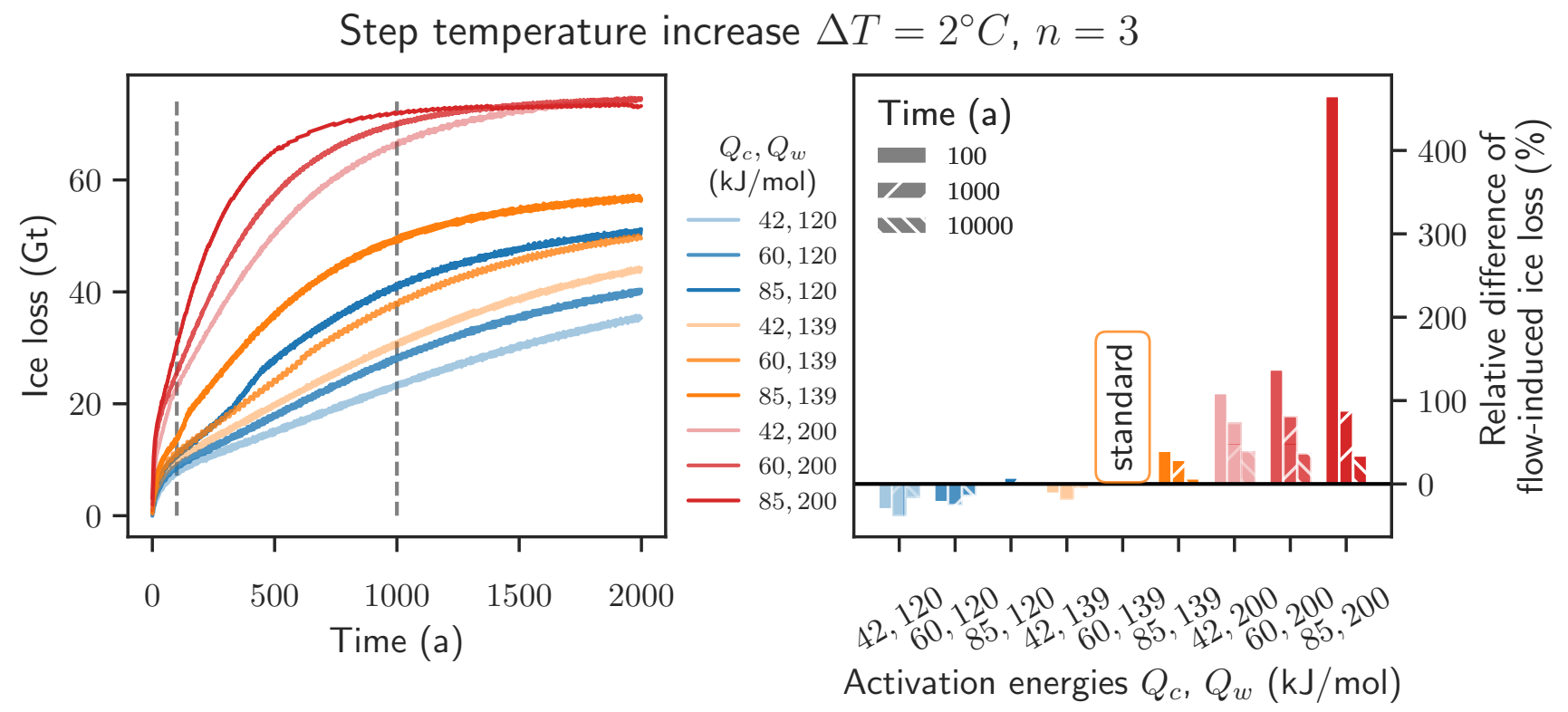

Figure S3. Effect of activation energy and flow exponent on flow-driven ice discharge with shallow ice and shallow shelf approximation: a) Mass of ice loss in a conceptual flowline setup, subject to a temperature anomaly forcing of $\Delta T=2^{\circ} \mathrm{C}$ and $n=3$. b) Relative difference of the volume of ice loss $d=\left(\Delta V / \Delta V_{0}-1\right)$ with ice loss with standard parameters as reference for the same conditions as a). Here the shallow shelf approximation is used for the sliding velocity while in other simulations sliding is inhibited. Compare to Figure ??, where only the shallow ice approximation (and thus no sliding) is taken into account. 